
\title{
is Research Suare \\ Understanding The Workforce That Supports Māori And Pacific Peoples With Type 2 Diabetes To Achieve Better Health Outcomes
}

Tania Mullane ( $\square$ tania.mullane@whitireia.ac.nz)

Whitireia Institute

Matire Harwood

University of Auckland Private Bag 92019

Isaac Warbrick

Auckland University of Technology Private Bag 92006

Taria Tane

University of Auckland Private Bag 92019

Anneka Anderson

University of Auckland Private Bag 92019

\section{Research Article}

Keywords: Diabetes, Indigenous, Qualitative, Māori, Pacifıc, Workforce

Posted Date: January 19th, 2022

DOl: https://doi.org/10.21203/rs.3.rs-1190090/v1

License: (1) This work is licensed under a Creative Commons Attribution 4.0 International License. Read Full License 


\section{Abstract}

Background: Prevalence of Type 2 diabetes mellitus (T2DM) is high among Māori and other Pacific Island peoples in New Zealand. Current health services to address T2DM largely take place in primary healthcare settings and have, overall, failed to address the significant health inequities among Māori and Pacific people with T2DM.

Culturally comprehensive T2DM management programmes, aimed at addressing inequities in Māori or Pacific diabetes management and workforce development, are not extensively available in New Zealand. Deliberate strategies to improve cultural safety, such as educating health professionals and fostering culturally safe practices must be priority when funding health services that deliver T2DM prevention programmes.

There is a significant workforce of community-based, non-clinical workers in South Auckland delivering diabetes self-management education to Māori and Pacific peoples. These include dietitians, community health workers and, more recently, kai manaaki (KM), but there is little information about these workers and their perspectives, challenges, effectiveness, and success in delivering these services.

This study aimed to understand perspectives and characteristics of KM and other community-based, non-clinical health workers, with a focus on how they supported Māori and Pacific Peoples living with T2DM to achieve better outcomes.

Methods: This qualitative study was undertaken underpinned by the Tangata Hourua research framework. Focus groups with dietitians, community health workers (CHWs) and KM took place in South Auckland, New Zealand. Thematic analysis of the transcripts was used to identify important key themes.

Results: Analysis of focus group meetings identified three themes common across the groups: whakawhanaungatanga (actively building relationships), cultural safety, and cultural alignment. However, there appeared to be two key differences for $\mathrm{KM}$ and $\mathrm{CHWs}$, who both preferred a multidisciplinary approach and described their experiences of feeling un/valued in their roles, when compared with dietitians. Generally, all three groups agreed that their roles required good relationships with the people they were working with and an understanding of the contexts in which Māori and Pacific Peoples with T2DM lived.

Conclusions: Supporting community based, non-clinical workers to build meaningful and culturally safe relationships with Māori and Pacific people has potential to improve diabetes outcomes.

\section{Background}

Globally, $8 \%$ of the world's population have type 2 diabetes mellitus (T2DM) [1] and significant increases are expected in developing countries [2-5]. Indigenous peoples, in particular, have poor outcomes from T2DM [6], including higher rates for complications such as eye, kidney, vascular disease and significantly advanced rate of mortality compared to non-Indigenous people [7]. T2DM is also a significant issue in Aotearoa New Zealand (hereafter referred to as NZ) with Māori, the Indigenous people of NZ, and Pacific Peoples experiencing significantly higher rates for diabetes and its complications [8]. Harwood and Tipene-Leach [9] go further, stating that 'For no other disease are significant health inequalities more obvious than when we look at diabetes.' ( $p$. 162). 
Community-centred care plays an important role in addressing these inequities through the provision of lifestyle advice, diabetes and self-management education, and medication [10]. In NZ a range of workers, including general and nurse practitioners, nurses, community health workers and dietitians, provide these services. Further efforts to improve access to Māori and Pacific Peoples have tended to focus on outreach by conventional services such as nurse clinics [11] and dietitians [12]. However, these may not be effective in achieving equitable outcomes for Māori and Pacific Peoples with T2DM. Further, there is little information about what kind of workforce, or workforce development, is required to improve diabetes outcomes for Māori and Pacific Peoples.

In 2016 the National Hauora Coalition (NHC), a Māori-led primary health organisation, received funding to test Mana Tū, an innovative programme for Māori and Pacific Peoples with T2DM. Mana Tū (which means 'to stand with authority'), a whānau ora approach to long-term conditions, is a Māori-led, Kaupapa Māori approach to supporting people living with poorly controlled T2DM, and their wider whānau (family members) [13]. Kai manaaki (KM) are skilled case managers who are embedded within a primary care service as core workforce; their case management approach supports diabetes self-management with Māori and Pacific Peoples living with poorly controlled T2DM [14].

In this paper we explore opportunities to better understand the KM workforce and compare their experience with other community-based, non-clinical diabetes workers. A key objective of this research is to use the findings to inform future strategies for Māori and Pacific health workforce development, to improve the delivery of services and diabetes outcomes for Māori and Pacific Peoples. This study aims to understand similarities and differences between KM and other community-based, non-clinical health workers, with a focus on how they supported Māori and Pacific Peoples living with T2DM to achieve better outcomes.

\section{Methods}

A qualitative study was undertaken within the Tangata Hourua research framework, which was developed by members of this research team [15]. Tangata Hourua draws from Kaupapa Māori and pan-Pacific methodologies and values the upholding of the rights of Indigenous peoples to have their knowledge and culture embedded in research, whilst also giving voice to Pacific Peoples living in NZ with shared or connected whakapapa. A major strength of the model is its potential to be utilised in research that aspires to contribute positively to Māori and Pacific knowledge and wellbeing [15].

Specifically, the study aimed to gain an understanding of the experiences for the main groups of communitybased, non-clinical workers currently supporting Māori and Pacific Peoples and their whānau (families), rather than a broad representation of all health workers who deliver T2DM programmes. Focus groups were determined to be the most appropriate method to obtain this information, presenting an excellent opportunity to focus on a particular issue, privileging the subjective knowledge and life experiences of respondents [16]. Further, focus group methods align with important Māori and Pacific principles including mana-enhancement (acknowledging the contribution and expertise of each participant), mahitahi (co-operation between participants and researchers) and manaakitanga (reciprocity) by providing a collective forum for participants to share their own experiences and contribute to improving the services that they know intimately [17].

The focus groups were conducted with the three main workforce groups working in South Auckland, New Zealand. We chose this region for its ethnic profile, as $13 \%$ of the population identify as Māori, representing $24 \%$ 
of all Māori in New Zealand [18], and 64\% of New Zealand's Pacific population currently lives in Auckland, representing $15 \%$ of the Auckland population [18]. The three main workforces, KM, community health workers (CHWs) and dietitians, participated in three separate focus groups. Potential participants were recruited by sending an invitation to organisations employing dietitians, $\mathrm{CHWs}$ and $\mathrm{KM}$. Once we had received more than six responses from one of the three workforces, we arranged focus group discussions which lasted 60-70 minutes and took place at work sites and at times that suited participants. Food and refreshments were provided for each group discussion. Informed consent and confidentiality agreements were obtained from all participants at the start of each meeting, and demographic information was collected (see Table 1).

The guiding questions were:

1. What can you tell me about your role?

2. What works well in your role and why?

3. What could be improved? And how?

4. What do you think is important to consider in working with Māori and Pacific Peoples living with T2DM?

5. How do you know if you are effective?

The primary researcher transcribed the recorded focus group discussions verbatim. Themes arising from the data were analysed, as informed by the Tangata Hourua model. This informed analysis places core Māori and Pacific values and concepts as a pou (pillar) to guide data analysis and theming. This is to ensure the authentic voices of Māori as Indigenous to New Zealand and the Pacific Peoples of Te Moana Nui a Kiwa (the Pacific Ocean) can be heard.

\section{Results}

Table 1 presents the number and demographic profile of participants for each focus group. The focus groups reflected the fact that women make up the great majority of the New Zealand health care workforce [19], with all participants being female. Analysis of the data identified common themes: whakawhanaungatanga (actively building relationships), cultural safety and cultural alignment. However, there were also two key differences for $\mathrm{KM}$ and $\mathrm{CHWs}$ who (1) preferred a multi-disciplinary team (MDT) approach, and (2) described their experiences of feeling un/valued in their roles, when compared with dietitians. The themes are presented here.

Table 1 - Profile of Focus Groups 


\begin{tabular}{|llll|}
\hline Focus Group & Number & Demographic Profile of the Focus Groups & \\
\cline { 3 - 4 } & & Ethnicity - self- identified & Gender \\
\hline Kai Manaaki & Seven & $\begin{array}{l}\text { Six Māori } \\
\text { One Indo-Fijian }\end{array}$ & $\begin{array}{l}\text { All } \\
\text { female }\end{array}$ \\
\hline $\begin{array}{l}\text { Community Health } \\
\text { Workers }\end{array}$ & Eight & Two Māori & All female \\
& & $\begin{array}{l}\text { Two Pacific Peoples (Cook Island Māori and } \\
\text { Samoan) }\end{array}$ & \\
\hline Dieticians & Four New Zealand European & All \\
& Seven & $\begin{array}{l}\text { Three Pacific Peoples (Tongan and Cook Island } \\
\text { Māori) }\end{array}$ & \\
\hline & Four New Zealand European & female \\
\hline
\end{tabular}

\section{Whakawhanaungatanga}

Whakawhanaungatanga is a fundamental principle which Mead [20] explains as reaching beyond family connections to establish and maintain personal and culturally relevant connections. All the participants concurred in stating that whakawhanaungatanga was a key component in strengthening and establishing knowledge of self, whānau and the community. They felt this was essential not just for the individual, but for whānau connections too, in that it created a safe space to share their lived experience of having T2DM.

The KM's perspective on whakawhanaungatanga illustrated the core premise of the concept:

"Trust building is important and how we build trust to them and then they tell things to us. ... treating my patients like they are my sister, my brother my whānau which quickly establishes a relationship."

"Talking about their families and about them first before diving into medical stuff ... build up a relationship, establishing a really good rapport, it's connection. ... Trust building is important and how we build trust to them and then they tell things to us; which allows clients to feel safe."

"We [Pacific Peoples] are more like a group rather than self ... [in] understanding them and also understand how the Pacific and Mãori live and what mattered to them, fitting into their world view" - Dietitian, Pacific.

They felt strongly that more could be done to highlight the importance of whakawhanaungatanga when working with Māori and Pacific Peoples and communities.

Whakawhanaungatanga has become a core fundamental of all the participants' professional roles. This was spoken about by New Zealand European participants as a strategy used specifically to build culturally meaningful connections and relationships when working with Māori and Pacific Peoples and their whānau.

"Things like whakawhanaungatanga is vital, nobody ever writes about it, its intrinsic to this community" - CHW, NZ European. 
"I think potentially as a non-Māori and non-Pacific there's a barrier there right at the start in terms of 'am I going to listen, am I going to understand ... mistrust with what's happened before' so how can we start to engage them?" - Dietician, NZ European.

There was also reflection on how a cultural approach was essential for non-Māori, non-Pacific workers if genuine relationships were to be built so as to achieve better diabetes outcomes for clients and their whānau:

"quite a few years ago I would have thought that was like such bad practice ... now I think it's so important, because I don't need a diet history to be able to do my job and do it well. So yeah, just massive changes of thought and process around how you do things and then obviously that's that for another time at building relationships ... sometimes I'll spend an entire session just chatting [in order to know each other].

\section{A culturally safe workforce}

Delivering services in traditional settings such as marae (traditional meeting houses) for Māori and church for Pacific Peoples were effective for shifting power to clients, due to their experience of cultural affirmation. One $\mathrm{CHW}$ described the importance of traditional cultural settings or principles for services for Māori and Pacific Peoples:

"Being based on the whenua [land] of a marae is key to what we do and how we do it. I don't think we can say that any other clinic in the whole of Auckland has as higher Māori ethnicity as we do. [It's a space] where they can just be and interact with others and be heard" - CHW, Māori.

"Being on the marae and having the marae support, working within an organisation that is guided by kaupapa Māori values ... it's privilege" - KM, Māori.

Participants based in clinics in non-traditional settings, such as community-based marae (Māori meeting house), spoke about how this contributed to a culturally safe work environment. They strongly advocated that being based on the marae was beneficial not only to them as part of the health team but also, more importantly, to the clients and their whānau who accessed the services there.

"Being based on the whenua of a marae is key to what we do and how we do it. I don't think we can say that any other clinic in the whole of Auckland has as higher Māori ethnicity as we do."

Those who were based at the marae saw it as an example of best practice, and saw that other providers looked to them as a role model and a centre of excellence. The clinic is becoming a hub for the community:

"The clinic works like a centre of the community, so people feel like they can pop in anytime, walk in clinics to see someone; There is also a spiritual component ... the idea of community, the idea of inclusion" - CHW, Māori.

\section{"These are my brothers and sisters"}

The importance of language was explained:

"I find the language that you use has to be simple ... non-judgmental lens, taking that judgement out, because they are already feeling so crap ... we don't use jargon from the clinic we use everyday language that we/they 
know. It's about creating a supportive environment for people to thrive in and that's also very important" - KM, Māori.

However, this required time. The concept of 'time for equity' allowed appointments to go for an hour or longer to "show you value them and their time". When we "just chill out listen, then they talk". Consequently, clients described feeling heard, and were more likely to:

"actually, come up with the solution you don't have to do anything, don't make assumptions that we know any more than they do they know" - KM, Māori.

"sometimes our appointments would go for an hour or so, 'cause they just needed that time to sort of warm up and then really really get out what they were trying to say or wanted to know ... there's a valuing of their time and what they're talking about ... it's a two-way thing it's not that anyone is better but because we had the time just sit there and listen and you know" - KM, Māori.

Cultural concordance - knowing the context within which Māori and Pacific Peoples with T2DM lived - was associated with better engagement and, subsequently, better outcomes. As one worker said, "these are my brothers and sisters. I know what works and what doesn't."

All groups felt that there were inadequate culturally specific resources to work with: "trying to find pictures [of healthy food options] that represent Māori or Pasifika people is really hard sometimes" with dietitians commenting that most resources were "very white" (CHW, NZ European).

Workers encouraged each other to know and incorporate their clients' 'normal day life' into interventions, as these encouraged clients to engage with the programme. Participants agreed that this was easier for Māori and Pacific workers because they lived similar lives and were:

“Understanding [of] everybody's work timetable, kids' timetable, how are we going to put your food and medication around what you are already doing, and how can we adjust that for your whole family's benefit. Go out to [local food] markets and understand where they shop and what food they eat" - CHW, NZ European.

All agreed that building Māori and Pacific workforce capacity in T2DM management was important as it allowed the workforce that was needed to reflect the Māori and Pacific population who had the highest incidence of T2DM. The dieticians, especially, strongly advocated that any workforce development should attract and retain more Māori and Pacific into health professional roles, which also supported the District Health Board goals of increasing more Māori and Pacific staffing.

Every one of the New Zealand European dieticians spoke about their self-awareness of unconscious biases, with one dietician acknowledging that some clients did not want to work with them because they were Pākehā (New Zealand European), which forced them to reflect on awareness and responsibility of cultural safety. This suggests all clinicians, regardless of their background or culture, may not be appropriate with anybody who walks through the door. A New Zealand European CHW also recognised the importance of a culturally appropriate person, of Pacific working with Pacific, as there was only one Pacific $\mathrm{CHW}$ in their organisation:

"Being in the community and working with Pacific Peoples means language is important, so having a PI CHW makes such a difference with supporting us with language, but also with the engagement, she can engage with 
them in a way that I can't and that's good."

"So having someone who is Māori or Pacifica working in the team is essential. Just obviously having a [Pacific person] involved in the delivery of the programme, having someone from that ethnic background and can sort of relate. Having a [Pacific person] involved in the delivery of the programme, having someone from that ethnic background and can sort of relate to the kids, being the same age and being relevant."

There was certainty from non-Māori and non-Pacific in recognising that professional development in cultural safety was necessary, in addition to building the capacity of the Māori and Pacific workforce.

\section{Multidisciplinary team approach}

The KM and CHWs both worked as part of a multidisciplinary teams (MDTs) and felt that this was best for managing T2DM for Māori and Pacific Peoples. An MDT approach was strength-based in the sense that different members of the health team had different roles based on their strengths or expertise. They described the importance of working in partnership with non-health service providers, including social or housing services, which facilitated access to interventions that addressed the wider determinants for health, such as poverty. For example, one KM described supporting a client to study for and sit their driver's licence, which then enabled them to attend job interviews and finally attain employment.

The KM in particular felt that being part of a wider team meant that they contributed to the wider environment, and this had an impact on the health and wellbeing of the whole community. This influence occurred at multiple levels. For example, in the primary care clinics, they participated in team meetings, shared experiences and learnings about good practice, and advocated for clients and their whānau. One example was teaching clinicians (GPs and nurses) how they could do more to remove barriers to diabetes care. They knew where to locate or refer people for services and dismissed siloed care:

"I don't know anyone outside the marae because we are so used to using who [multiple health and social service workers] we have here" - KM, Māori.

In contrast, the dieticians did not mention a multidisciplinary approach and their focus group discussion concentrated just on their own role.

\section{Feeling un/valued}

CHWs described feeling marginalised by some diabetes services because they were not in formally recognised roles. KMs are also not regulated yet they had a very different experience. They described a workplace that provided regular supervision and opportunities for peer support, in addition to access to programmes that fulfilled self-determined professional development needs. They felt valued for their cultural and social determinant expertise, and that they were contributing beyond the service to people and their communities.

"Actually, we were always treated like professionals of our field" - KM, Māori.

"even smaller 'aha' moment such as they are smiling, they brought their daughter or someone with them to learn some more, sometimes a community of people sitting there waiting for you" - KM, Māori

Again, there was no mention of this from dietitians. 


\section{Discussion}

This study aimed to understand the experiences of the KM, the workforce for Mana Tū, compared with other community-based, non-clinical health workers, and how they support Māori and Pacific Peoples living with T2DM to achieve better outcomes. The analysis of focus group meetings identified similarities and differences between the perspectives of dietitians, $\mathrm{CHWs}$ and $\mathrm{KM}$. All three groups agreed that their roles required good relationships with the people they were working with, and an understanding of the contexts in which Māori and Pacific Peoples with T2DM lived. Further, there was an expectation that services were culturally safe, and required more Māori and Pacific workers and resources. However, CHWs and KM reported a preference for working as part of an MDT and described their experiences as unregulated health workers.

All participants placed high value on whakawhanaungatanga, which for them meant making of culturally meaningful connections with others. They felt this aligned with Māori and Pacific worldviews and cultural values in terms of engaging the collective rather than just individuals. Their perspectives strongly indicated that culturally appropriate communication and relationship building, such as whakawhanaungatanga, encouraged individuals and their whānau to be more open, form relationships and feel safe to discuss health and lives. The concept of whakawhanaungatanga is now a commonly understand term and integrated into health practice by Māori and non-Māori [21, 22], and should not be regarded as a one-off event to connect with the patient and whānau but, rather, something ongoing that is built upon whilst retaining safe boundaries [23].

In response to the unfair burden of health inequalities disproportionately affecting Māori and Pacific Peoples, there is an urgent need to create culturally safe environments [24]. An Indigenous response integrates, culturally safe practices and concepts into health service delivery $[25,26]$. Achieving cultural safety requires an acknowledgement of one's own culture, and personal biases in order to understand better the culture of others who are different to oneself, with the intention to further recognise and respect the cultures of people, whānau and communities [27]. In New Zealand, a much-needed evolution from cultural competency to cultural safety is occurring and supports the historical call by Māori seeking self-governing health care, which is strongly based on their health status and rights with respect to the Treaty of Waitangi $[22,28]$. The enablement of culturally responsive healthcare organisations and workforce development [29] is a principal strategy towards the goal of improved cultural competence in health services and systems that better meet the needs of Māori and Pacific Peoples [30]. There are strong calls for this not only to permeate health organisations, but to be sector-wide, and to include social services, education, justice, and research, using systematic and sustainable approaches [26]. An accountable mechanism for providing culturally safe care, as defined by patients and their communities, measured through progress towards achieving health equity [27] is something that may be addressed by the recently announced health reforms. These reforms will establish a Māori Health Authority, with the express aim of enhancing Māori rangatiratanga (governance) for Māori over hauora (health) with greater influence ensured throughout the entire health system [28].

The non-regulated workforce is a priority area needing people whose knowledge and skills are required to respond to the culturally responsive models of care needed to meet the health demands of Māori and Pacific and support to better health outcomes [10]. 'Kaiāwhina' is the over-arching term used to describe non-regulated roles in the health and disability sector such as $\mathrm{CHW}$ s and $\mathrm{KM}$ with essential qualities of resilience, diversity, skill and commitment to support hauora (health) outcomes for all in Aotearoa New Zealand (50\% being Māori) [31]. 
It is important to note that being non-clinical or non-regulated does imply a lack of knowledge or professionalism, as these roles bring the crucial cultural, local and interpersonal skills and expertise that are integral to building relationships and trust with the whānau and communities they work within. A study by Boulton et al. [32] illustrated the restricted career advancement opportunities for Māori CHWs due to the lack of formal role recognition and limited training options. In contrast, the KM described the importance of feeling valued in their roles when they were members of the MDT, received professional development opportunities and had regular peer review. Research suggests that when physicians and non-physician professionals cohesively work together, improved patient outcomes, including better clinical outcome measures and higher patient satisfaction, occur [33]. Considering the recent health reforms in NZ, including the development of a health charter and its focus on a relevant health workforce for communities [30,34], there is scope to support CHWs and $\mathrm{KM}$ in the ways described here.

\section{Conclusion}

This study is a first of its kind, with unique findings from an investigation into three distinct roles for health staff who work with Māori and Pacific with T2DM. There was deliberate approach to undertake research that was strength-based, with a focus on the system rather than individual people and their behaviour. Importantly, as much as this was about the workers, the ultimate objective was on achieving excellent outcomes for Māori and Pacific Peoples. However, there are several limitations to this study. This is a small project with three focus groups in one area of NZ, and therefore it is not generalisable to other settings or workforce groups. However, it does provide rich insights into the experiences of those people working with an important cohort and has the potential to inform workforce development strategies both here in NZ and for other Indigenous communities.

Indigenous comprehensive health and social programmes that achieve better diabetes outcomes for Māori and Pacific communities require the right workforce. Our study suggests that support for community-based, nonclinical workers to build meaningful and culturally safe relationships with communities, and contribute as valued team members, will improve diabetes outcomes.

\section{Glossary}




\begin{tabular}{|ll|}
\hline Aotearoa & New Zealand \\
\hline Hauora & Health \\
\hline Kai Manaaki & Health navigator \\
\hline Kaiāwhina & Community health worker \\
\hline Kaupapa Māori & Māori world view \\
\hline Mahitahi & Do-operation between participants and researchers \\
\hline Mana enhancing & Acknowledging the contribution and expertise of each participant \\
\hline Mana Tū & Stand with authority \\
\hline Māori & Indigenous people of New Zealand \\
\hline Marae & Traditional meeting house of Māori \\
\hline Rangatiratanga & Governance \\
\hline Tangata Hourua & Combined people \\
\hline Te Moana-nui-a-kiwa & Indigenous Pacific peoples of the wider Pacifıc Ocean \\
\hline Treaty of Waitangi & Founding document in New Zealand between Māori and the Crown \\
\hline Whakapapa & Family/genealogy \\
\hline Whakawhanaungatanga & Actively building relationships \\
\hline Whānau & Family \\
\hline
\end{tabular}

\section{Declarations}

\section{Ethics approval and consent to participate}

Ethics approval was obtained, on the $23^{\text {rd of }}$ June 2020 by the Auckland University of Technology Ethics Committee. reg no. 20/8. Informed consent to participate in the study was obtained from all participants prior to their involvement and all methods performed were in accordance with relevant guidelines and regulations.

\section{Consent for publication}

The authors declare that they consent to publish

\section{Availability of data and materials}

All ethics and research guidelines used for this article are publicly available.

Under Indigenous data sovereignty this data is protected and permission to share transcripts was not sought.

\section{Competing interests}


The authors declare that they have no competing interests.

\section{Funding}

No funding was received for this research project.

\section{Authors Information}

Tania Mullane, Bachelor of Nursing Pacific, Whitireia Institute, DX Box: SX33459, Porirua, Wellington, New Zealand.

Matire Harwood, Department of General Practice and Primary Care, Faculty of Medical and Health Sciences, University of Auckland Private Bag 92019, Auckland Mail Centre, Auckland 1142, New Zealand

Isaac Warbrick, Taupua Waiora Research Centre, Faculty of Health and Environmental Sciences, Auckland University of Technology Private Bag 92006, Auckland 1142, New Zealand

Taria Tane, Department of General Practice and Primary Care, Faculty of Medical and Health Sciences, University of Auckland Private Bag 92019, Auckland Mail Centre, Auckland 1142, New Zealand

Anneka Anderson, Faculty of Medical and Health Sciences, Te Kupenga Hauora Māori, University of Auckland Private Bag 92019, Auckland Mail Centre, Auckland 1142, New Zealand

\section{Authors' contributions}

TM and $\mathrm{MH}$ designed the study. TM undertook recruitment, interviewing and transcription. TM analysed the data and led the drafting of the manuscript with $\mathrm{MH}$ and IW contributing to these steps. All authors contributed to the writing and review of the manuscript and all authors have approved the final manuscript.

\section{Acknowledgements}

The authors wish to acknowledge the participants of the focus groups for contributing their time, sharing their views and stories.

\section{References}

1. Campbell JA, Walker RJ, Dawson AZ, Egede LE. Prevalence of diabetes, prediabetes, and obesity in the Indigenous Kuna population of Panamá. J Racial Ethn Health Disparities. 2019 Aug;6(4):743-51. https://doi.org/10.1007/s40615-019-00573-0.

2. Young TK, Schraer CD, Shubnikoff EV, Szathmary EK, Nikitin YP. Prevalence of diagnosed diabetes in circumpolar indigenous populations. Int J Epidemiol. 1992 Aug 01;21(4):730-36. https://doi.org/10.1093/ije/21.4.730.

3. Zimmet P, Alberti KG, Shaw J. Global and societal implications of the diabetes epidemic. Nature. 2001 Dec;414(6865):782-7. https://doi.org/10.1038/414782a.

4. King M, Smith A, Gracey M. Indigenous health part 2: the underlying causes of the health gap. The Lancet. 2009 Jul 04;374(9683):76-85. https://doi.org/10.1016/S0140-6736(09)60827-8. 
5. Catherine HY, Zinman B. Type 2 diabetes and impaired glucose tolerance in aboriginal populations: a global perspective. Diabetes Res Clin Pr. 2007 Nov 01;78(2):159-70. https://doi.org/10.1016/j.diabres.2007.03.022.

6. Naqshbandi M, Harris SB, Esler JG, Antwi-Nsiah F. Global complication rates of type 2 diabetes in Indigenous peoples: A comprehensive review. Diabetes Res Clin Pr. 2008 Oct 01;82(1):1-7. https://doi.org/10.1016/j.diabres.2008.07.017.

7. Cundy T, Drury P. Rising tide of type 2 diabetes in younger people. What can primary care do? Bpac Diabetes Toolbox. 2001. Available from: https://bpac.org.nz/2021/diabetes-younger.aspx.

8. Ministry of Health. About diabetes. 2020. Available from: https://www.health.govt.nz/our-work/diseasesand-conditions/diabetes/about-diabetes.

9. Harwood M, Tipene-Leach D. Diabetes. In: Robson B, Harris R, editors, Hauora: Māori Standards of Health IV. A study of the years 2000-2005. Wellington: Te Roopu Rangahau Hauora a Eru Pomare; 2007, p. 160-67. Available from: .

10. Ministry of Health. Living well with diabetes: a plan for people at high risk of or living with diabetes 20152020. 2015. Available from: https://www.health.govt.nz/publication/living-well-diabetes.

11. New Zealand Guidelines Group. Guidance on the management of type 2 diabetes. Wellington: New Zealand Guidelines Group; 2011.

12. Howatson A, Wall C, Tūrner-Benny P. The contribution of dietitians to the primary health care workforce. J Prim Health Care. 2015;7(4):324-32. https://doi.org/10.1071/hc15324.

13. Harwood M, Tane T, Broome L, Carswell P, Selak V, Reid J, et al. Mana Tū: a whānau ora approach to type 2 diabetes. NZ Med J. 2018;131(1485). Available from: https://journal.nzma.org.nz/journal-articles/mana-tua-whanau-ora-approach-to-type-2-diabetes.

14. Selak V, Stewart T, Jiang Y, Reid J, Tane T, Carswell P, et al. Indigenous health worker support for patients with poorly controlled type 2 diabetes: study protocol for a cluster randomised controlled trial of the Mana Tū programme. BMJ Open. 2018;8(12):1-9. https://doi.org/10.1136/bmjopen-2017-019572.

15. Mullane T. Tangata Hourua Framework. Paper presented at: 2021 Indigenous Wellbeing Conference; 6-7 December 2021; Cairns. Available from:

https://astmanagement.eventsair.com/QuickEventWebsitePortal/iwc/iwcprogram/Agenda/AgendaltemDetail?id=7368dd0d-4810-452a-910f-6cc8d4a7c08c.

16. Colucci E. On the use of focus groups in cross-cultural research. In: Liamputtong, P, editor, Doing crosscultural research. Dordrecht: Springer; 2008, p. 233-52. https://doi.org/10.1007/978-1-4020-8567-3_15.

17. Pihama L, Cram F, Walker S. Creating methodological space: A literature review of Kaupapa Maori research. Can J Native Educ. 2002 Jan 01;26(1):30-43.

18. Statistics New Zealand. Māori population estimates: At 30 June 2017. 2017. Available from: https://www.stats.govt.nz/information-releases/maori-population-estimates-at-30-june-2017.

19. Ministry of Health. Health workforce development: An overview. 2006. Available from: https://www.health.govt.nz/system/files/documents/publications/health-workforce-dev.pdf.

20. Mead HM. Tikanga Māori: living by Māori values. Revised ed. Wellington: Huia; 2016.

21. Moorfield JC. Te Aka Online Māori-English, English-Māori dictionary and index. n.d. Available from: https://maoridictionary.co.nz/ 
22. Keelan W. Whanaungatanga is for everyone. Health Safety and Quality Commission New Zealand. 2019. Available from: https://www.hqsc.govt.nz/blog/whanaungatanga-is-for-everyone.

23. Lacey C, Huria T, Beckert L, Gilles M, Pitama S. The Hui Process: a framework to enhance the doctor-patient relationship with Māori. NZ Med J. 2011;124(1347):72-8. Available from:

https://journal.nzma.org.nz/journal-articles/the-hui-process-a-framework-to-enhance-the-doctor-patientrelationship-with-maori.

24. DeSouza R. Wellness for all: the possibilities of cultural safety and cultural competence in New Zealand. J Res Nurs. 2008;13(2):125-35. https://doi.org/10.1177\%2F1744987108088637.

25. Tiatia J. Pacific cultural competencies: a literature review. Ministry of Health. 2008. Available from: https://www.moh.govt.nz/notebook/nbbooks.nsf/0/1E4A8D37EAF49FA4CC25744A000A599C/\$file/pacificcultural-competencies-literature-review-feb08.pdf

26. Curtis E, Jones R, Tipene-Leach D, Walker C, Loring, B, Paine SJ, et al. Why cultural safety rather than cultural competency is required to achieve health equity: a literature review and recommended definition. Int J Equity Health. 2019;18:174. https://doi.org/10.1186/s12939-019-1082-3.

27. Wepa D, editor. Cultural safety in Aotearoa New Zealand. Melbourne: Cambridge University Press; 2015.

28. Laing P, Pomare E. Māori health and the health care reforms. Health Policy. 1994;29(1-2):143-56. https://doi.org/10.1016/0168-8510(94)90012-4.

29. Jongen C, McCalman J, Bainbridge, R. Health workforce cultural competency interventions: a systematic scoping review. BMC Health Serv Res. 2018;18:232. https://doi.org/10.1186/s12913-018-3001-5.

30. Department of the Prime Minister and Cabinet. The new health system. 2021. Available from: https://dpmc.govt.nz/our-business-units/transition-unit/response-health-and-disability-systemreview/information.

31. Ministry of Health. Kaiāwhina workforce plan for the health and disability workforce 2020-2025. 2021. Available from: https://kaiawhinaplan.org.nz.

32. Boulton, AF, Gifford, HH, Potaka-Osbourne, M. Realising Whānau Ora through community action: the role of Māori community health workers. Education for Health. 2009;22(2):188. Available from: Realising whānau ora through community action: the role of Māori community health workers - PubMed (nih.gov)

33. Grumbach K, Bodenheimer T. Can health care teams improve primary care practice? JAMA. 2004;291(10):1246-1251. https://doi.org/10.1001/jama.291.10.1246.

34. New Zealand Government. About the health reforms. The future of health. 2021. Available from: https://www.futureofhealth.govt.nz/about-the-reforms. 